\title{
Range perception through apparent image speed in freely flying honeybees
}

\author{
M.V. SRINIVASAN, ${ }^{1}$ M. LEHRER, ${ }^{2}$ W.H. KIRCHNER, ${ }^{3}$ AND S.W. ZHANG ${ }^{4}$ \\ 'Centre for Visual Sciences, Research School of Biological Sciences, Australian National University, Canberra, Australia \\ ${ }^{2}$ Abtl. Neurobiologie, Zoologisches Institut der Universitaet Zuerich, Winterthurerstrasse - 190 , \\ CH-8057 Zurich, Switzerland \\ ${ }^{3}$ Zoologisches Institut der Universitaet, Lehrstuhl fuer Tierphysiologie, D-8700 Wuerzburg, Germany \\ ${ }^{4}$ Institute of Biophysics, Academia Sinica, Beijing, Peoples Republic of China
}

(Received November 1, 1990; ACCePted January 9, 1991)

\begin{abstract}
When negotiating a narrow gap, honeybees tend to fly through the middle of the gap, balancing the distances to the boundary on either side. To investigate the basis of this "centering response," bees were trained to fly through a tunnel on their way to a feeding site and back, while their flight trajectories were filmed from above. The wall on either side carried a visual pattern. When the patterns were stationary vertical gratings, bees tended to fly through the middle of the tunnel, i.e. along its longitudinal axis. However, when one of the gratings was in motion, bees flying in the same direction as the moving grating tended to fly closer to it, while bees flying in the opposite direction tended to fly closer to the stationary grating. This demonstrates, directly and unequivocally, that flying bees estimate the distances of surfaces in terms of the apparent motion of their images. A series of further experiments revealed that the distance to the gratings is gauged in terms of their apparent angular speeds, and that the visual system of the bee is capable of measuring angular speed largely independently of the spatial period, intensity profile, or contrast of the grating. Thus, the motion-sensitive mechanisms mediating range perception appear to be qualitatively different from those that mediate the well-known optomotor response in insects, or those involved in motion detection and ocular tracking in man.
\end{abstract}

Keywords: Distance, Depth, Optical flow, Apparent motion, Vision, Insect flight

\section{Introduction}

The eyes of most insects are comparatively close together, and lack focusing mechanisms, so that they must use cues other than stereopsis or accommodation to gauge the distances of objects in the environment. It has been suggested that moving insects perceive the distance of objects in terms of the speeds of their images on the retina, a higher image speed being associated with a smaller range (Wallace, 1959; Collett, 1978; Eriksson, 1980; Goulet et al., 1981; Collett \& Harkness, 1982; Horridge, 1986; Cheng et al., 1987; Collett, 1988; Lehrer et al., 1988; Srinivasan et al., 1989). Recently, Sobel $(1990 a, 1990 b)$ confirmed Wallace's (1959) original finding that locusts use a peering motion of the head to infer object range.

We recently demonstrated that honeybees flying along a tunnel tend to fly through the middle by balancing the apparent motions of the images of the walls on the two sides (Kirchner \& Srinivasan, 1989). The present study explores this "centering response" quantitatively, in an attempt to discover the parameters of image motion that are used to extract range informa-

Reprint requests to: M.V. Srinivasan, Centre for Visual Sciences, Research School of Biological Sciences, Australian National University, P.O. Box 475, Canberra, A.C.T. 2601, Australia. tion, and to probe the mechanisms by which these parameters are measured. In particular, we compare the roles played by two parameters: (1) contrast frequency and (2) apparent image speed.

The well-studied optomotor response in insects is mediated by a motion-detecting mechanism that is sensitive to contrast frequency (review by Reichardt, 1987). Such a system is, however, incapable of measuring image speed unambiguously, and independently of the structure of the image (review by Borst \& Egelhaaf, 1989). On the other hand, a system that measures the apparent speed of an image independently of its structure would be capable of providing a reliable estimate of range.

In the present study, we examine how range estimation is influenced by apparent image speed and contrast frequency, by presenting freely flying bees with a variety of situations in which these two parameters are placed in competition.

\section{Methods}

\section{Experimental technique}

The experimental apparatus was a tunnel formed by two walls $40 \mathrm{~cm}$ long, $20 \mathrm{~cm}$ high, and $12 \mathrm{~cm}$ apart (Fig. 1). Each wall consisted of a sheet of rubber stretched around a pair of drums. 


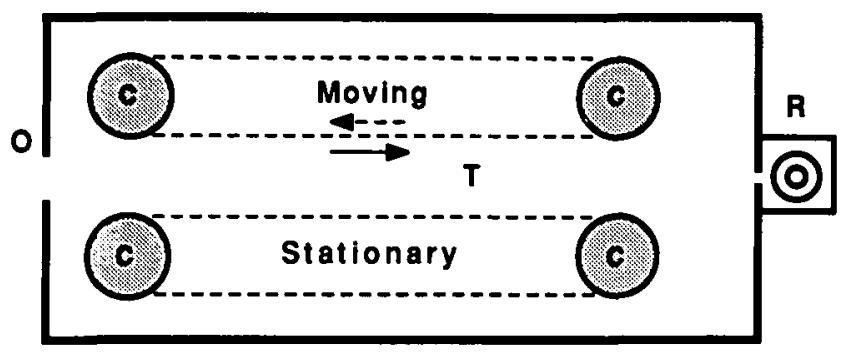

B

Fig. 1. Schematic, overhead view of experimental apparatus (not to scale). Bees are trained to enter the perspex box B through the opening $\mathrm{O}$ and to fly along tunnel $\mathrm{T}$ to receive a reward of sugar water in the box $R$. Each side wall of the tunnel consists of a sheet of rubber stretched around a pair of drums (C). Gratings or other patterns are affixed to the sheets. A motor coupled to one of the drums enables the pattern on one of the walls to be moved in either direction (arrows) at any desired speed.

Gratings or other patterns (described below) were affixed to the sheets. A d.c. motor coupled to one of the drums enabled the pattern on one of the walls to be moved in either direction (arrows, Fig. 1) at any desired speed.

Groups of 4-6 freely flying honeybees (Apis mellifera) were marked and trained to enter the apparatus, fly along the tunnel, and receive a reward of sugar solution in a box at the end of the tunnel (Fig. 1). After the bees had received ca. 60 rewards, their flight trajectories were video-filmed from above as they flew along the tunnel in either direction (i.e. toward the reward, or homeward after having been rewarded). Depending upon the experiment, both walls were stationary or one of them was in motion (Fig. 1). In some experiments, sheets of transparent perspex were placed in front of the walls to prevent the flight trajectories from being influenced by air currents generated by the moving patterns. However, since experiments conducted without these sheets revealed no obvious differences, data from both kinds of experiments were pooled.

The patterns on the walls usually consisted of a vertically oriented, one-dimensional, black-and-white grating with an intensity profile that was square-wave, random, sinusoidal, or sawtooth-shaped. In some experiments, one of the walls carried a sheet of white (photocopying) paper, or gray paper of the same mean luminance and spectral composition as the gratings.

The square-wave and random gratings, of contrast 0.85 , were constructed by glueing strips of black paper onto a background consisting of white (photocopying) paper. The squarewave gratings had periods of $2.5 \mathrm{~cm}, 3.5 \mathrm{~cm}, 7 \mathrm{~cm}$, and $10 \mathrm{~cm}$. The random grating was constructed similarly, except that the widths of successive black bars and intervening white spaces were random, integral multiples of $0.4 \mathrm{~cm}$, determined by the random-number generator on a computer. The sinusoidal and sawtooth-profile gratings were created using a computer-driven laser printer, capable of generating 64 gray levels in square pixels of size $0.7 \mathrm{~mm}$. These patterns were carefully screened for defects arising from toner deficiencies in the laser printer. Screening was done by visual inspection, as well as through intensity-profile measurements made using a video camera coupled to a computer-driven frame grabber. The sinusoidal gratings had periods of $1.5 \mathrm{~cm}, 3 \mathrm{~cm}$, or $6 \mathrm{~cm}$. The Michelson contrasts used $\left[\left(I_{\max }-I_{\min }\right) /\left(I_{\max }+I_{\min }\right)\right]$, as derived from the measured intensity profiles] were $0.60,0.15$, or lower than 0.05 (nominally, homogeneous gray). The sawtooth-profile gratings had a period of $3.0 \mathrm{~cm}$, and a contrast of 0.75 .

The (angular) spatial frequencies of the gratings, as seen by a bee flying along the axis of the tunnel, ranged from 0.01-0.04 cycle/deg for the square-wave gratings, $0.017-0.07 \mathrm{cycle} / \mathrm{deg}$ for the sinusoidal gratings, and $0.035 \mathrm{cycle} / \mathrm{deg}$ for the sawtooth-profile gratings. The random grating possessed a spectrum of spatial frequencies distributed over the range $0-0.13$ cycle/deg. Our prior knowledge of the spatial acuity of the honeybee's visual system (e.g. Srinivasan \& Lehrer, 1988) indicates that all of the gratings used in these experiments should have been clearly resolvable.

\section{Analysis of flight paths}

Flight trajectories, recorded at 25 frames/s by the camera and video recorder, were subsequently analyzed frame-by-frame to measure average positions along the width of the tunnel, average flight velocities, etc. This was done either manually - by plotting successive positions of bees on a sheet of transparent acetate paper affixed to the screen of the video monitor - or by superimposing the video image onto a digitizing tablet using a half-reflecting mirror. Detailed histograms of bee positions, as shown in Figs. 2 and 3, were obtained by analyzing the digitized data on a computer to determine the relative frequency with which bees were found in each cell of an array of $20 \times 10$ cells. (This array represented a total area of $26 \times 12 \mathrm{~cm}^{2}$ ). These relative frequencies are depicted as an array of squares, the linear dimension of each square being proportional to frequency. In other instances (as in the data presented in Figs. 4-7, and Fig. 11), each trajectory was assigned a single mean position along the width of the tunnel, by averaging the $y$ coordinates of bee positions digitized along a central $26-\mathrm{cm}$ segment of the trajectory. In some instances (Figs. 4 and 11 ), the mean positions of several trajectories were then averaged to obtain a mean trajectory position and a standard deviation about this mean. Velocities of bees, and of pattern movement, were obtained by measuring the distance travelled over a known number of frames. Knowing these and the position of the trajectory along the tunnel width, the apparent angular speeds of the patterns on the eyes of the flying bee could be calculated using the formulae shown in Fig. 5.

\section{Results}

When the two walls of the tunnel are stationary and carry identical gratings, bees tend to fly through the middle of the tunnel (on average), as shown in Fig. 2a. The mean position of the flight trajectories along the width of the tunnel is not significantly displaced from the axis of the tunnel ( $t$-test, $P>0.30$ ). How were the bees gauging and balancing the distances to the gratings on the two sides? One possibility is that they were balancing the speeds of the retinal images of the gratings on the two eyes. To test this possibility, we examined flight trajectories when one of the gratings was in motion. When this grating moved at a constant speed in the direction of the bees' flight thereby reducing the velocity of retinal image motion on that eye relative to the other eye - the bees' trajectories shifted toward the side of the moving grating (Fig. 2b). However, when the grating moved in a direction opposite to that of the bees' flight - thereby increasing the speed of retinal image motion on 

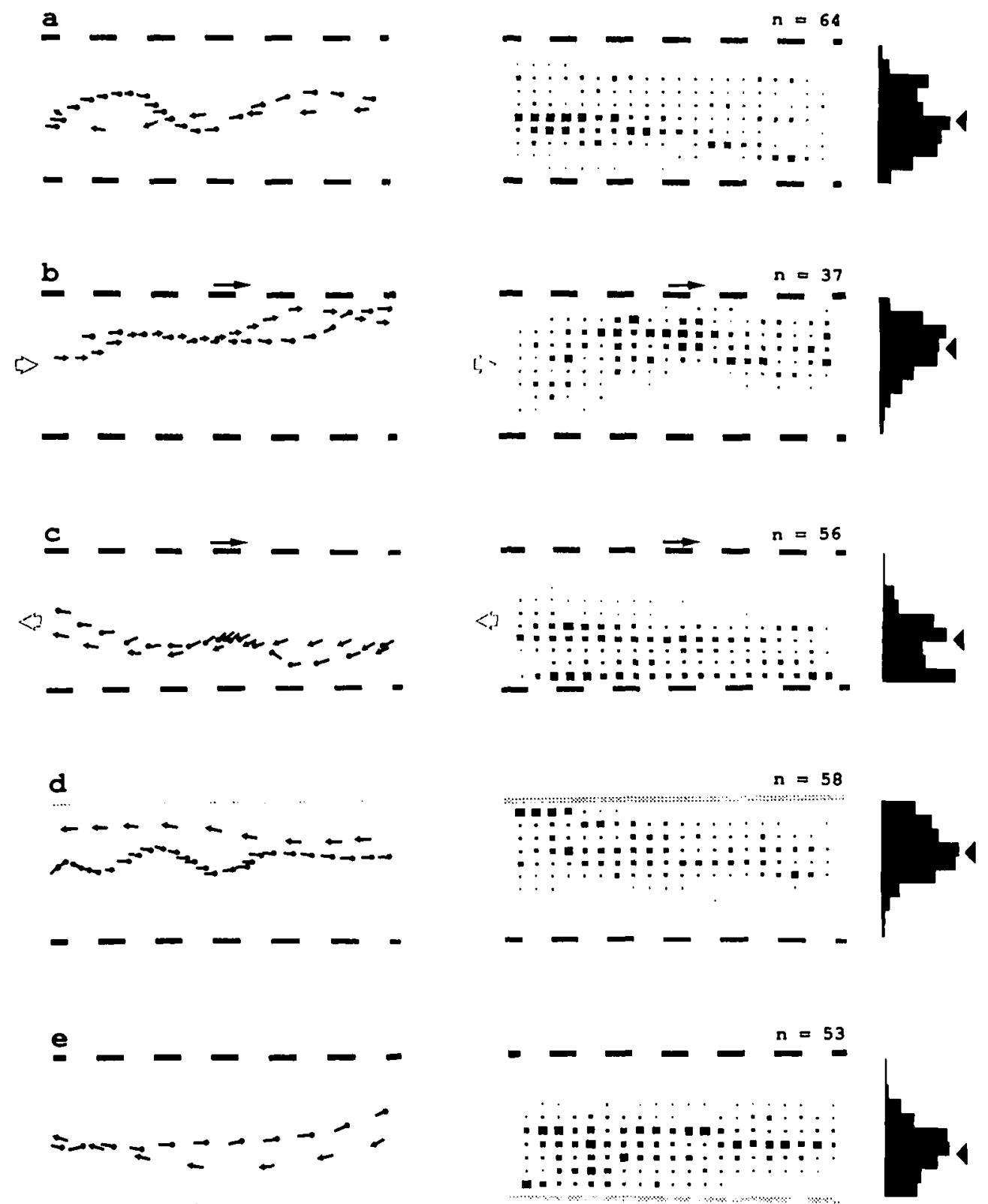

Fig. 2. The centering response: Experient demonstrating that flying bees use apparent image motion to estimate range. Bees were trained to fly along a tunnel, in which the side walls carried vertically oriented square-wave gratings of period $3.5 \mathrm{~cm}$. Flights were filmed from above at 25 frames/s with (a) stationary gratings on either side, (b) one grating moving at a speed of $20 \mathrm{~cm} / \mathrm{s}$ in the direction of the bees' flight, (c) this grating moving at $20 \mathrm{~cm} / \mathrm{s}$ in a direction opposite to that of the bees' flight, and (d,e) the moving grating replaced by a homogeneously grey sheet. The left-hand column shows two typical trajectories (depicted by the two different bee-symbols) in each case. The right-hand column shows the spatial distributions of the bees' positions, obtained by analyzing a number of trajectories in each case, as described in Methods. Flights in both directions were analyzed in (a), (d), and (e), while flights in the directions of the large arrows were analyzed in (b) and (c). The histograms on the extreme right depict the distribution of the bees' trajectories along the width of the tunnel, averaged over the central, 26-cm segments that were analyzed (see Methods). The arrowhead next to each histogram depicts the mean value of the distribution. $n=$ number of trajectories analyzed in each case.

that eye relative to the other eye - the trajectories shifted away from the side of the moving grating (Fig. 2c). Finally, when one of the gratings was replaced by a homogeneously gray sheet of paper and the other grating was stationary, the trajectories shifted toward the homogeneous side (Fig. 2d,e). There is a significant difference between the average locations of the flight trajectories, measured along the width of the tunnel, between the experimental conditions corresponding to Figs. 2a,b; 2a,c; $2 \mathrm{~b}, \mathrm{c} ; 2 \mathrm{a}, \mathrm{d} ; 2 \mathrm{a}, \mathrm{e}$; and $2 \mathrm{~d}$,e ( $t$-test, $P<0.001$ for all cases).

These findings suggest that when the walls are stationary, the bees maintain equidistance by balancing the apparent speeds of the retinal images in the two eyes. A slower motion on one eye is evidently taken to imply that the grating on that side is further away, causing the bee to fly along a trajectory closer to it; 


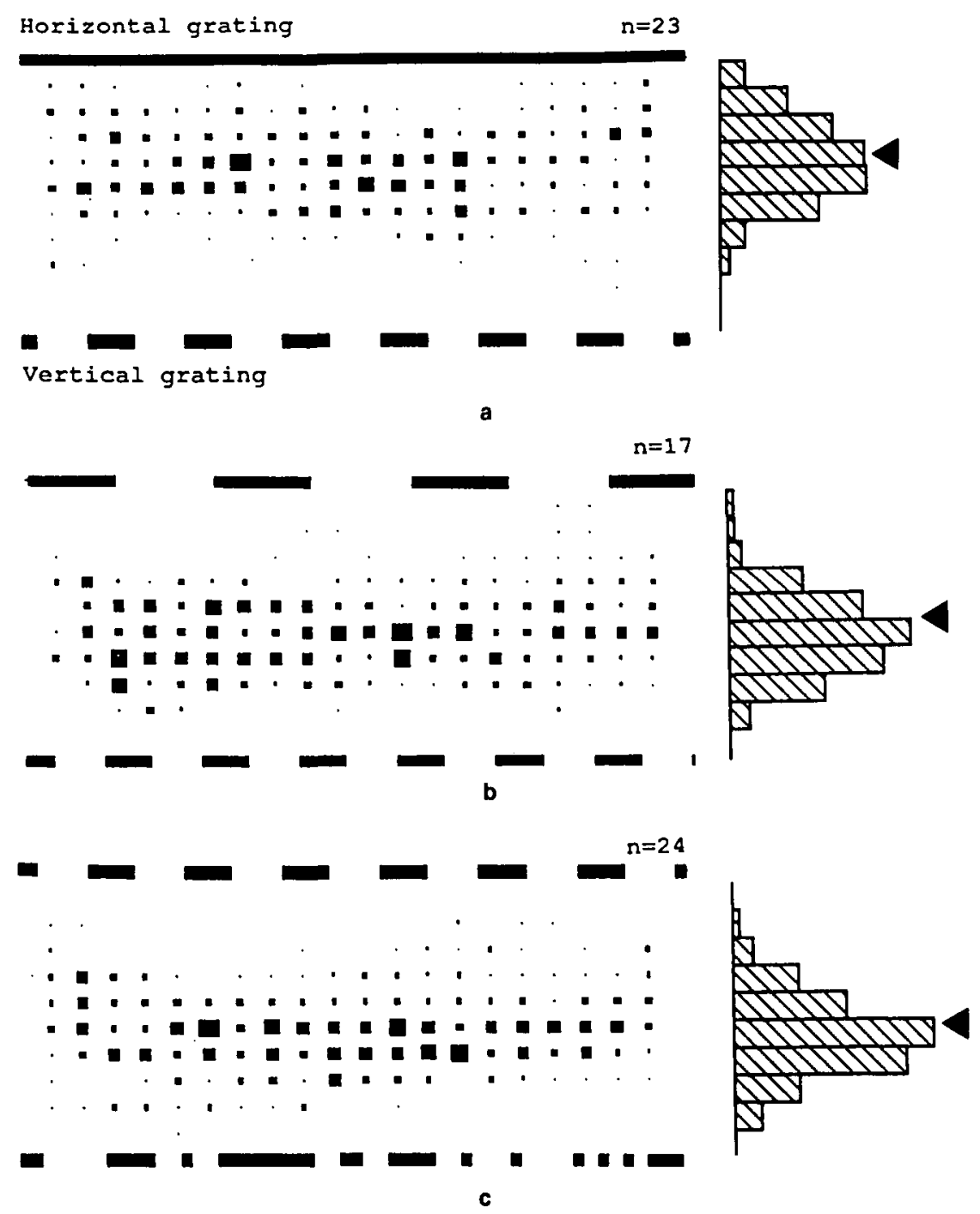

Fig. 3. Distribution of bees' positions in the tunnel when the two walls carry different, stationary patterns. Flights in both directions were analyzed. In all three cases, the pattern on one wall is a vertically oriented square-wave grating of period $3.5 \mathrm{~cm}$. In (a), the pattern on the other wall is a horizontally oriented square-wave grating of the same period. In (b), it is a vertically oriented square-wave grating of period $7.0 \mathrm{~cm}$. In (c), it is a spatially random grating with characteristics as described in Methods. In (b) and (c), the mean position of the bees' trajectories (arrowhead) is not displaced significantly from the tunnel axis, whilst in (a), bees fly significantly closer to the wall carrying the horizontal grating. Histograms have the same meaning as in Fig. 2. Comments in text. $n=$ number of trajectories analyzed in each case.

a faster motion of the image, on the other hand, has the opposite effect. When one eye sees homogeneous grey, it receives no movement stimulus and the bees consequently fly closer to that side.

\section{What parameters of image motion mediate the centering response?}

The most likely interpretation of the results described so far is that the flying bees are trying to balance the apparent angular speeds, in the horizontal plane, of the images on the two eyes as they fly along the tunnel. There is, however, another possibility, namely, that they infer the distances to the two walls by moving alternately toward one wall and then the other (i.e. zigzagging along the tunnel) and comparing the looming (rate of expansion) of the patterns on the two walls. Although this seems unlikely (from the relatively straight profiles of the flight trajectories), we tested for it by analyzing the flight trajectories of bees flying through the tunnel when both walls were stationary, with one wall carrying a horizontal grating, and the other wall a vertical one. Although looming cues can be obtained from the horizontal grating as well as the vertical grating, horizontal motion cues would be provided only by the vertical grating. Therefore, we would expect bees to fly close to the axis of the tunnel if they use looming-motion cues, but close to the horizontal grating if they use horizontal-motion cues. The results (Fig. 3a) show that the bees fly distinctly closer to the horizontal grating: the mean position of the trajectories deviates significantly from the tunnel axis $(P<0.0005)$. Thus, the importance of looming cues seems negligible. This conclusion is supported 


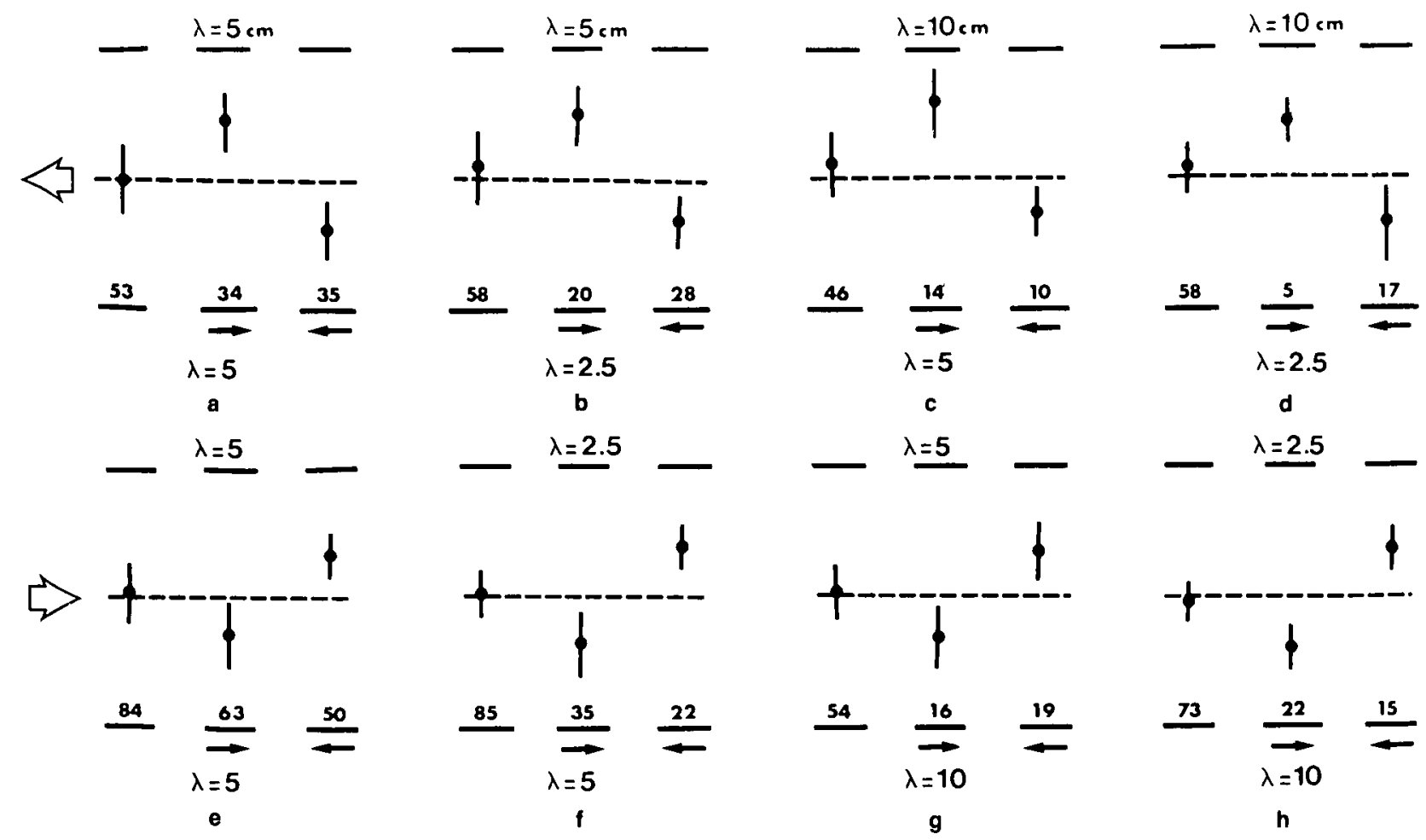

\begin{abstract}
Fig. 4. Contrast-frequency invariance of the centering response: Analysis of flight trajectories for a number of different combinations ( $a-h)$ of vertically oriented square-wave gratings on the two walls. Each of the eight panels shows the mean and the standard deviation of the positions of the flight trajectories for a particular combination of gratings, of period $\lambda$, when both gratings are stationary (left-hand side of each panel), or when one of the gratings moves at a constant speed of $27.5 \mathrm{~cm} / \mathrm{s}$ toward the reward box (center), or the exit (right), as shown by the small arrows. The panels (a-d) represent flights toward the exit, whilst the panels (e-h) represent flights toward the reward box (as shown by the large arrows). In all cases, the mean position of the trajectories does not deviate significantly from the tunnel axis when both gratings are stationary, but does so in the appropriate direction (as in Fig. 2) when one of the gratings is in motion. The numbers represent the number of flights analyzed in each case.
\end{abstract}

by the finding that the position of the flight trajectories is strongly affected by horizontal motion of a vertical grating (Figs. 2b,c). If the centering response were mediated primarily by looming-motion cues, one would not expect the response to be affected by the superposition of a horizontal component of motion, as this would not affect the looming component.

\section{Dependence of the centering response on contrast frequency}

The experiments described so far leave open the possibility that the bees were comparing the temporal frequencies of the intensity fluctuations ("contrast frequencies") produced by the successive dark and light bars of the gratings, as seen by the individual photoreceptors of the two eyes, rather than the apparent angular velocities of the patterns on the two walls. It is important to consider this possibility because it is now well-established that optomotor behavior - the best known motiondriven response in insects - is a response that depends upon contrast frequency, rather than angular speed (reviews by Reichardt, 1987; Borst \& Egelhaaf, 1989).

To test the importance of contrast frequency in the centering response, we analyzed the trajectories of bees flying along the tunnel when the two walls carried stationary gratings, but with the grating on one side having a spatial frequency twice that on the other (Fig. 3b). Despite the fact that this configuration produced twice the contrast frequency on one eye as on the other, the mean position of the bees' trajectories did not deviate significantly from the axis of the tunnel (Fig. $3 \mathrm{~b} ; P>0.4$ ), indicating that contrast frequency is not an important parameter in the centering response. In a further experiment, again performed with stationary patterns on the two walls, one wall carried a periodic grating whilst the other carried a grating that was spatially random, comprising a broad range of spatial frequencies (details in Methods). Here again, the bees' trajectories were located close to the tunnel axis (Fig. $3 c ; P>0.05$ ), indicating that, as far as the centering response is concerned, the temporal pattern of stimulation of individual photoreceptors is not crucial to the measurement of apparent motion.

The role of contrast frequency in the measurement of apparent motion was examined more extensively in a series of experiments using a number of different combinations of spatial frequencies for the gratings on the two walls. The results, shown in Fig. 4, indicate that, as long as both gratings are stationary and resolvable by the eye, the bees' trajectories are centered on the axis of the tunnel regardless of the spatial frequencies of the gratings on the two sides. The insensitivity of the centering response to spatial frequency (and therefore contrast frequency) is evident even when the spatial frequencies 


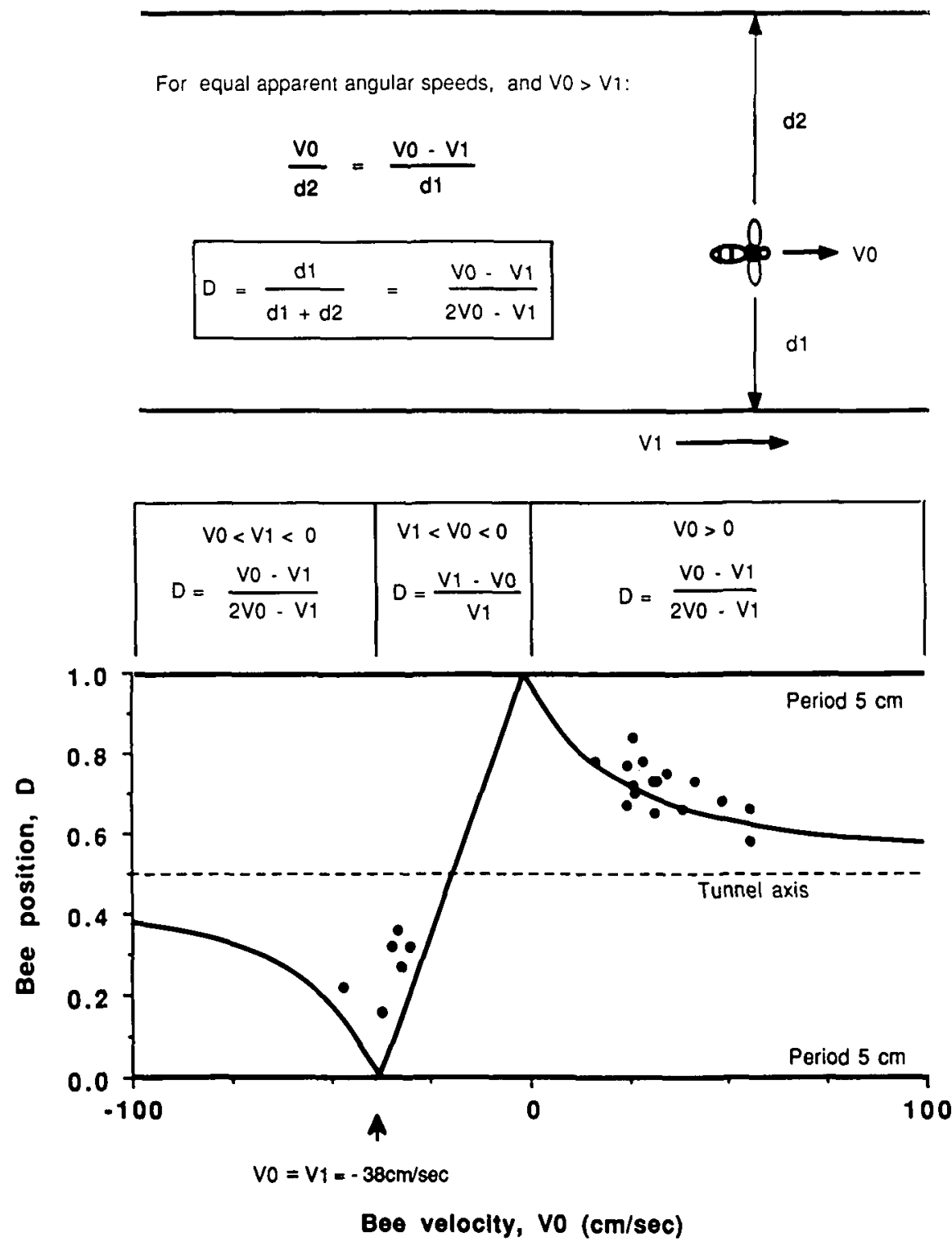

Fig. 5. Dependence of the centering response on apparent angular velocity: Experiment testing the hypothesis that bees balance the apparent angular speeds of the images on the two eyes as they fly through a tunnel in which one wall carries a stationary grating, and the other a moving one. If the hypothesis is true, then for any bee velocity $V 0$ and grating velocity $V 1(=-38 \mathrm{~cm} / \mathrm{s}$ in this particular case), it should be possible to predict the position of the trajectory along the width of the tunnel, as explained in the text and specified in the upper panel. The lower panel compares the theoretically predicted positions (continuous curve) with those experimentally observed (dots). Each wall carries a vertically oriented square-wave grating of period $5 \mathrm{~cm}$.

of the gratings on the two sides differ by a factor of as much as four [Fig. 4, see left-hand segments of panels (d) and (h)]. However, when one of the gratings is in motion (Fig. 4, see center and right-hand segments of each panel), the bees' flight trajectories are clearly displaced from the tunnel axis $(P<0.0025$ in all cases). The direction of displacement then depends consistently upon whether the grating moves with or against the direction of the bees' flight. The measurements with the moving gratings constitute an important control, because they demonstrate (1) that the bees are indeed attending to the apparent motion of the gratings on the two sides as they fly along the tunnel; and (2) when the gratings on the two sides are stationary, the resulting centering response is not simply a consequence of sat- uration of the responses of the movement-sensitive mechanisms in the two eyes.

In summary, the results of the above experiments suggest that contrast frequency is not an important parameter in the measurement of the apparent motion of the patterns. It is therefore very likely that apparent angular speed is, by default, the relevant parameter.

\section{Dependence of the centering response on apparent angular speed}

It is possible to test more directly whether the bees are indeed measuring angular speed, as follows. If the flying bees are try- 


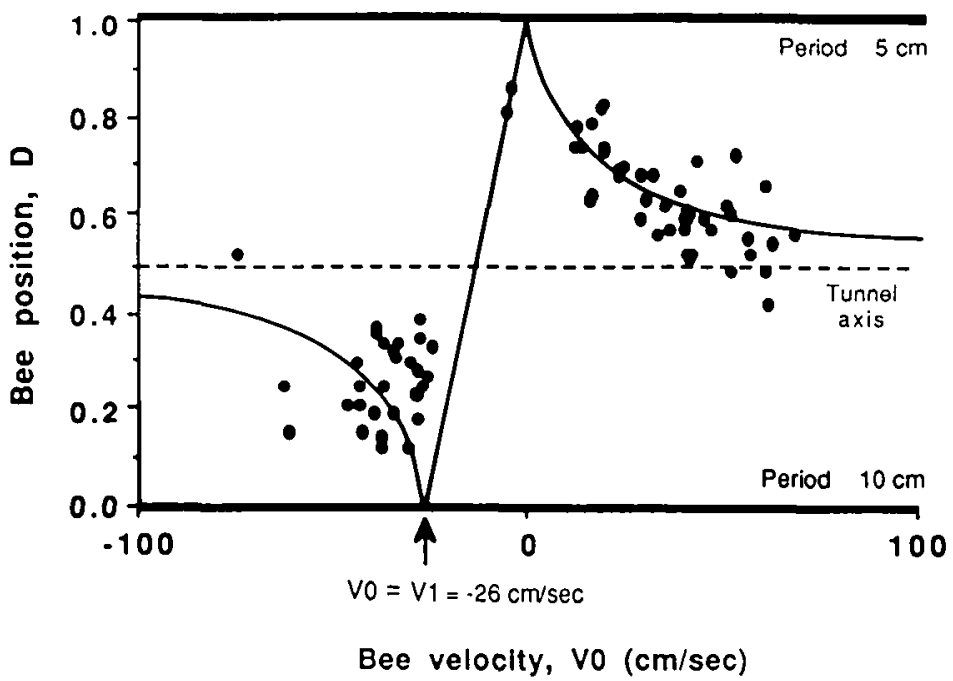

Fig. 6. Experiment similar to that shown in Fig. 5, but with gratings of different periods on the two walls. In this case, the 5-cm grating is stationary while a 10-cm grating moves at a constant velocity of $26 \mathrm{~cm} / \mathrm{s}$. Symbols are as in Fig. 5 .
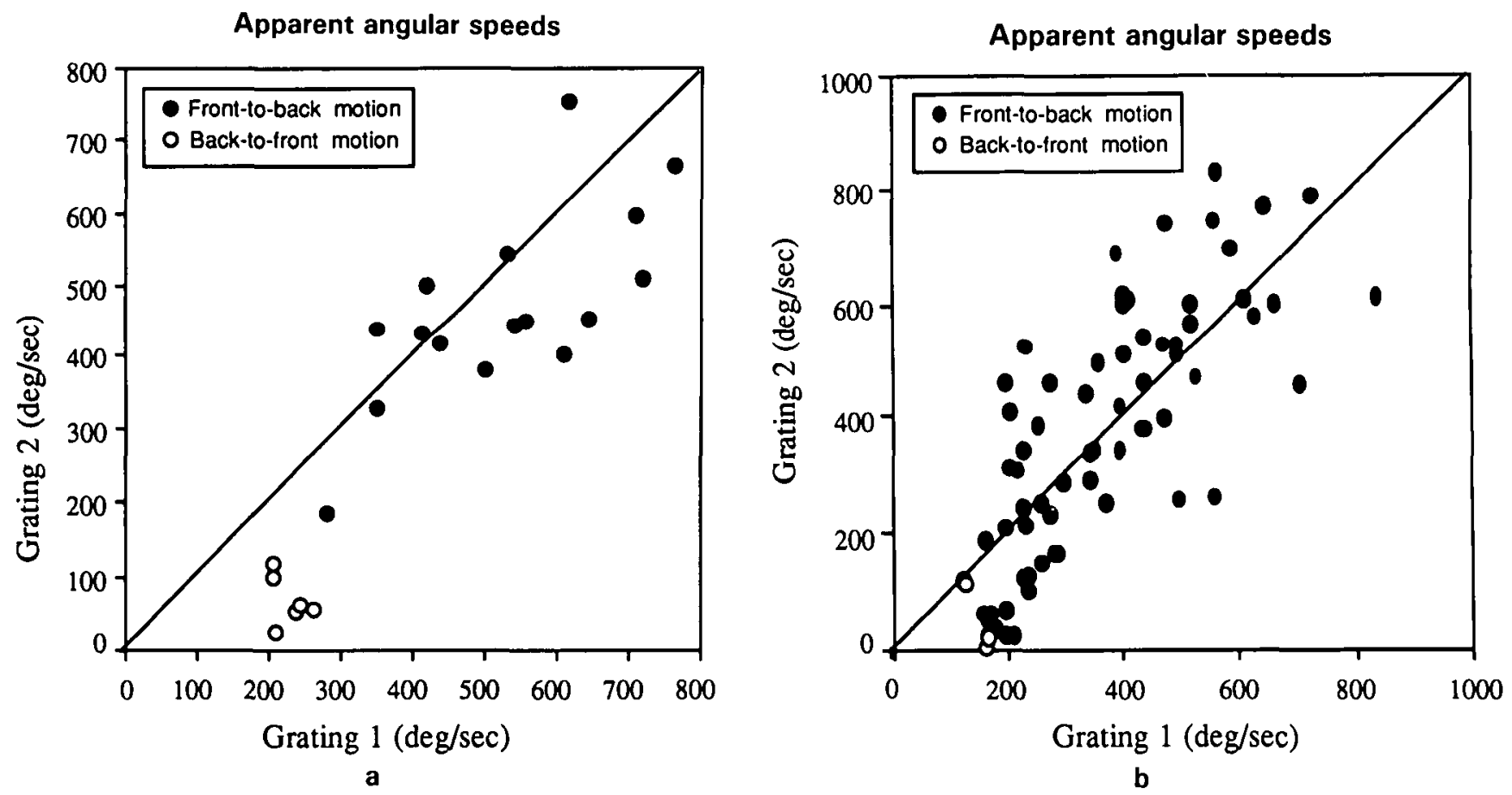

Fig. 7. Comparison of apparent angular velocities experienced by the two eyes: Panel a shows a comparison of the apparent angular speeds experienced by the bees' eyes for each of the flights analyzed in Fig. 5. The filled circles represent flights in a direction opposite to that in which the grating moves, while the open circles represent flights in the same direction, but at a lower speed, so that one grating appears to move in the back-to-front direction on the eye. Panel $b$ shows a similar comparison for the flights analyzed in Fig. 6.

ing to balance the apparent angular speeds of the patterns as seen by the two eyes, then when one of the patterns is in motion and we know its linear speed as well as that of the bee, we should be able to predict where, along the width of the tunnel, the bee's trajectory should be located so that the apparent angular velocities of the two patterns are equal. With reference to Fig. 5, we see that, for a bee flying at a speed $V 0$, and a pat- tern moving in the same direction with a linear speed $V 1$, equality of apparent angular speeds requires that

$$
\begin{aligned}
& V 0 / d 2=(V 1-V 0) / d 1, \quad \text { when } V 0<V 1 \\
& V 0 / d 2=(V 0-V 1) d 1, \quad \text { when } V 0 \geq V 1
\end{aligned}
$$


and that $V 0 / d 2=(V 0+V 1) / d 1$ when the bee flies in a direction opposite to that in which the pattern moves.

The above expressions can be rewritten to evaluate the quantity $d 1 /(d 1+d 2)$, which is the theoretically expected distance of the trajectory from the wall carrying the moving pattern, expressed as a fraction of the width of the tunnel. Denoting this quantity by $D$, we obtain

$$
\begin{aligned}
& D=(V 1-V 0) / V 1, \quad \text { when } V 0<V 1 \\
& D=(V 0-V 1) /\left(2 V_{0}-V_{1}\right), \quad \text { when } V 0 \geq V 1
\end{aligned}
$$

and

$$
\begin{aligned}
& D=(V 0+V 1) /(2 V 0+V 1) \\
& \text { when } V 0 \text { is oppositely directed to } V 1 .
\end{aligned}
$$

The results of an experiment designed to test these predictions are shown in Fig. 5. Here each wall carries a square-wave grating of period $5 \mathrm{~cm}$. The grating on one wall is stationary, whilst that on the other wall (the lower wall in the figure) moves at a constant velocity $V 1=-38 \mathrm{~cm} / \mathrm{s}$. (The negative sign denotes motion towards the entrance.) The continuous curve shows the theoretically expected relationship between bee speed ( $V 0$, abscissa) and bee distance ( $D$, ordinate), for bees flying with or against the direction of motion of the moving grating. The dots depict the experimentally measured relationship between the same two variables. Each dot represents one trajectory, where $V 0$ was measured as the average velocity of the bee (in $\mathrm{cm} / \mathrm{s}$ ), and $D$ was measured as the mean distance of the trajectory from the moving pattern, expressed as a fraction of the tunnel width. It is evident that the measurements fit the theory quite well, especially in the case of bees flying in the direction opposite to that of grating motion $(V 0>0)$.

The point $D=0$ on the theoretical curve corresponds to a situation in which the bee and the pattern are moving in the same direction at the same speed. In this situation, the moving grating does not move relative to the bee's eye. To the bee, therefore, this grating would be indistinguishable from a grating that is stationary and infinitely far away. One would thus expect that bees flying at the same speed and in the same direction as the moving grating would fly very close to it; the results (Fig. 5) show that this is indeed the case.

Consider now a bee flying at different speeds along the axis of the tunnel, while one of the gratings is in motion. As the speed of the bee decreases toward zero, the eye viewing the stationary grating would experience a progressively lower angular speed, and this would be a progressively smaller fraction of the angular speed experienced by the other eye. This is true irrespective of whether the bee flies with or against the moving grating. Therefore, with decreasing flight speed $(|V 0| \rightarrow 0)$, we would expect the position of the trajectory to shift progressively closer to the stationary grating $(D \rightarrow 1.0)$. The data (Fig. 5) show that this is indeed what occurs. On the other hand, as the speed of a bee flying along the tunnel axis increases $(|V 0| \rightarrow$ infinity), the ratio of angular speeds seen by the two eyes would approach unity, irrespective of the bee's direction of flight. Therefore, with increasing flight speed we would expect the position of the trajectory to approach the axis of the tunnel asymptotically $(D \rightarrow 0.5)$. The data show that this is indeed what occurs in the case of bees flying opposite to the direction of motion of the grating. Corresponding data for bees flying in the same direction as the grating are not available, as very few bees are found to fly at the requisite speeds.

The results of an experiment similar to that described above (see Fig. 5), but using gratings of different spatial frequencies on the two walls, are shown in Fig. 6. Here one wall carried a stationary grating of period $5 \mathrm{~cm}$, while the other carried a grating of period $10 \mathrm{~cm}$ moving at a speed of $26 \mathrm{~cm} / \mathrm{s}$. Again, it is evident that the data agrees reasonably well with the theoretically expected relationship between $D$ and $V 0$, calculated on the assumption that the flying bee balances the apparent angular speeds of the gratings on the two eyes, irrespective of their spatial structure.

A comparison of the apparent angular speeds seen by the two eyes, for each of the sets of flights analyzed in Figs. 5 and 6 , is shown in Fig. 7a and $7 \mathrm{~b}$, respectively. For flights in which both gratings appear to move in the front-to-back direction over the eyes (filled circles, Fig. 7a and $7 b$ ), the apparent angular speeds range from $c a .200 \mathrm{deg} / \mathrm{s}$ to $c a .800 \mathrm{deg} / \mathrm{s}$, depending upon the bee's flight speed and position along the width of the tunnel. It is evident that, in these flights, the bees tend to balance the apparent angular speeds on the two eyes, rarely allowing them to differ by more than $50 \%$.

Until now we have only considered situations in which each grating appears to move in the front-to-back direction over the corresponding eye. Let us now consider the case in which one grating appears to move in the front-to-back direction, whilst the other appears to move in the back-to-front direction. This situation - which would rarely be encountered during translatory flight in the real world-occurs when a bee flies in the same direction as the moving grating, but at a lower speed. In Figs. 5 and 6 , this corresponds to the domain of bee velocities for which $V 1<V 0<0$ (see the center panel above the graph in Fig. 5). Not many data points are available in this domain of bee velocities, because bees moving in the same direction as the grating tend to "catch up" with it, as indicated by the clustering of data points near $V 0=V 1$ in Figs. 5 and 6 (see arrow on the abscissa). Nevertheless, there are several data points, pertaining to this condition, that are in good agreement with the theoretical prediction (continuous curve) based on the assumption of equal apparent angular speeds on the two eyes. The apparent angular speeds experienced by the two eyes under these conditions are depicted by the open circles in Fig. $7 \mathrm{a}$ and $7 \mathrm{~b}$. Some but not all of the points fall close to the 45-deg line, confirming that there is a tendency to balance apparent angular speeds even under these unnatural conditions. The departure of some of the data points from the 45-deg line (see Fig. 7) may well be due to geometrical constraints imposed by the tunnel. For example, considering the data of Figs. 5 and 7a, a bee flying in the same direction as the moving grating and at the same speed $(38 \mathrm{~cm} / \mathrm{s})$ would experience zero angular speed on the eye viewing that grating, but it cannot experience an angular speed of less than $180 \mathrm{deg} / \mathrm{s}$ on the eye viewing the other (stationary) grating, owing to the finite width of the tunnel $(12 \mathrm{~cm})$.

In summary, the above findings indicate that bees flying through the tunnel tend to balance apparent image speeds even when the motions are oppositely directed on the two eyes. In other words, it is possible that the underlying motion-detecting 
mechanism is insensitive to the direction of movement (see also Discussion).

\section{What region of the eye is involved in measuring apparent angular speed?}

In calculating the theoretical curves for Figs. 5 and 6, we have assumed that the bee compares the apparent angular speeds in the lateral regions of the two eyes, i.e. along directions at right angles to the tunnel axis, where the velocity is a maximum. Is this really the case, or do the bees compare average velocities measured over a substantial fraction of the visual field of each eye? To investigate this question, we analyzed flight trajectories of bees when one wall was uniformly white, while the other wall carried a single, dark vertical stripe placed halfway along its length (Methods). The results show that the bees deflect sharply away from the stripe, but only when it approaches the lateral direction of view of the eye (Fig. 8). Many flights, not included in the analysis shown, actually involve more extreme deflections, ending in crashes against the blank wall and termination of flight. It is clear that the response induced by the stripe is not one of fixation, but, rather, avoidance. The shape of the trajectories suggest that angular speed is monitored chiefly by the lateral fields of view of the eyes, along directions approximately at right angles to that of flight. This concurs with Lehrer's (1990) recent observation that the lateral eye regions are the ones most sensitive to drift of the retinal image. An alternative interpretation of these trajectories would be that the centering response is associated with a fairly high threshold of apparent angular speed, which is exceeded only in the lateral fields of view. This possibility, however, is unlikely since the response is driven by apparent angular speeds that span a factor of ten (Fig. 7), and is detectable even in walking bees, where these speeds can be as low as $40 \mathrm{deg} / \mathrm{s}$ (data not shown).
Operating range of the centering response in terms of flight speeds, contrast frequencies, and apparent angular speeds

Histograms of the bees' flight speeds through the tunnel under various stimulus conditions are given in Figs. 9 and 10. When both gratings are stationary, flight speeds range from 10-70 $\mathrm{cm} / \mathrm{s}$ (Fig. 9). Depending on the spatial periods of the gratings on the walls, these speeds produce contrast frequencies ranging from $1-28 \mathrm{~Hz}$, and the contrast frequencies experienced by the two eyes in a given flight can differ by a factor as high as four. Assuming that the bees fly close to the tunnel axis (on average, as shown by the data in Fig. 4), the resulting apparent angular speeds experienced by the lateral eye regions range from $\mathrm{ca}$. 90$650 \mathrm{deg} / \mathrm{s}$ (Fig. 9).

Flight speeds are distributed somewhat differently when one of the gratings is in motion, as shown in Fig. 10. Here one wall carries a stationary grating of period $10 \mathrm{~cm}$, while the other carries a grating of period $2.5 \mathrm{~cm}$ moving at $27.5 \mathrm{~cm} / \mathrm{s}$. We already know that this stimulus elicits a clear centering response: these data were obtained from the same group of flights that was analyzed to obtain the results shown in Fig. 4 [panels (d) and (h)]. Bees flying in a direction opposite to that of the moving grating exhibit speeds ranging from $10-60 \mathrm{~cm} / \mathrm{s}$, experiencing contrast frequencies of $1-6 \mathrm{~Hz}$ from the stationary grating and 15-35 Hz from the moving grating (Fig. 10, left panel). Assuming that their flight trajectories are displaced from the tunnel axis such that the average value of the ratio $[d 1 /(d 1+d 2)]$ is 0.71 (as indicated by the data in panels (d) and (h) of Fig. 4), these bees would experience apparent angular speeds of $c a .160$ $\mathrm{deg} / \mathrm{s}$ to $c a .960 \mathrm{deg} / \mathrm{s}$ from the stationary grating and $c a .250$ $\mathrm{deg} / \mathrm{s}$ to $\mathrm{ca} .575 \mathrm{deg} / \mathrm{s}$ from the moving grating (Fig. 10, left panel). It is clear that the bees are able to balance approximately the apparent angular speeds on the two eyes even under conditions in which the contrast frequencies experienced by the two eyes differ by a factor of as much as ten. Bees flying in the same

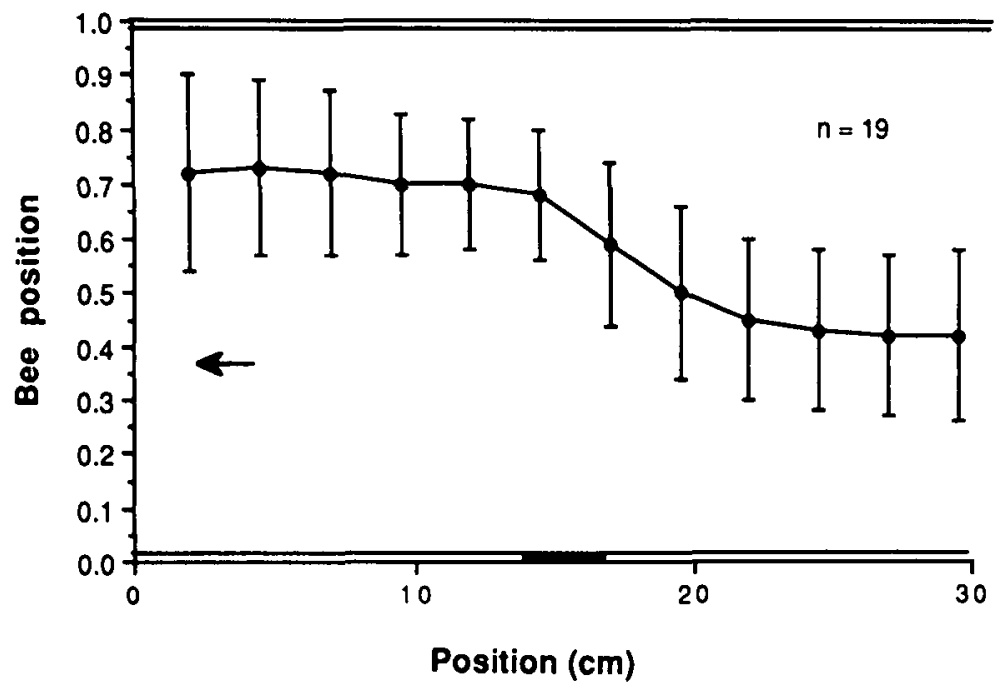

Fig. 8. Experiment designed to explore the region of the visual field that is involved in monitoring apparent angular speed. The figure shows the trajectory (depicted as the mean and standard deviation of $19 \mathrm{flights}$ ) when one wall is white and featureless, while the other carries a single, vertical dark stripe halfway along the tunnel, as shown. There is a sharp kink in the trajectory as the stripe passes the lateral field of view of the eye, suggesting that angular speed is monitored in a direction approximately at right angles to that of flight. 


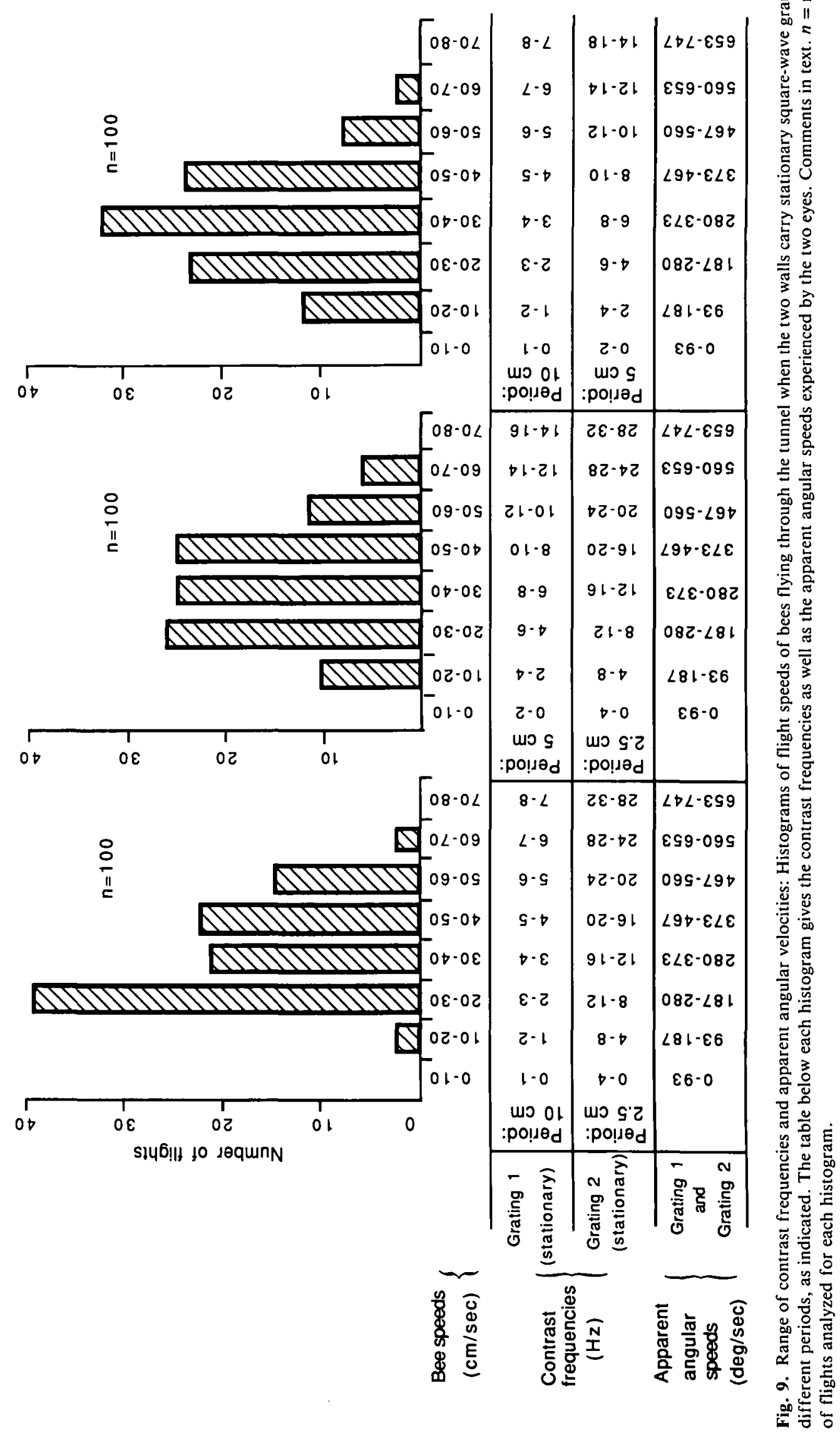



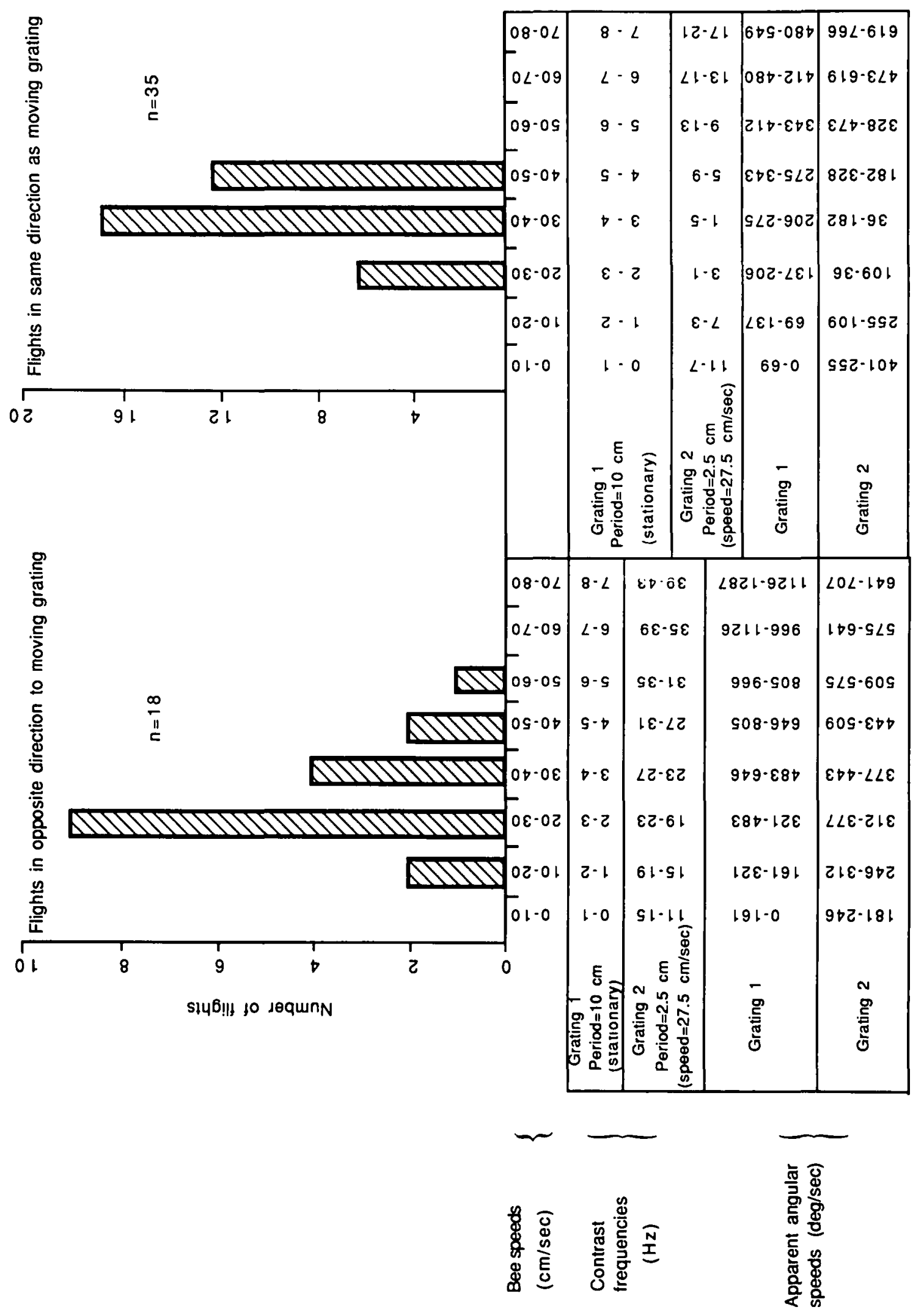
direction as the moving grating exhibit speeds ranging from 20 $50 \mathrm{~cm} / \mathrm{s}$, experiencing contrast frequencies of $2-5 \mathrm{~Hz}$ from the stationary grating and $0-9 \mathrm{~Hz}$ from the moving grating (Fig. 10, right panel). Assuming that their flight trajectories are displaced from the tunnel axis such that the average value of the ratio $[d 2 /(d 1+d 2)]$ is 0.68 (as indicated by the data in panels (d) and (h) of Fig. 4), these bees would experience apparent angular speeds ranging from $135-340 \mathrm{deg} / \mathrm{s}$ in the front-to-back direction from the stationary grating, and apparent angular speeds ranging from $\mathrm{ca} .105 \mathrm{deg} / \mathrm{s}$ in the back-to-front direction to $325 \mathrm{deg} / \mathrm{s}$ in the front-to-back direction from the moving grating (Fig. 10, right panel). It is evident that the moving grating influences the speed with which bees fly through the tunnel, causing them to fly slower when going in the opposite direction, and to avoid low speeds when flying in the same direction. At moderate grating speeds, bees going in the same direction as the moving grating seldom fly slower than the grating (Fig. 10, right panel), presumably to avoid the rather unnatural condition of apparent back-to-front motion on the eye during straight-ahead flight.

\section{Dependence of the centering response on the intensity profile of the pattern}

All of the patterns used in the experiments described so far consisted of black and white stripes. These patterns are characterized by regions of uniform intensity, "black" or "white," separated by boundaries where the intensity changes abruptly from one level to the other. Since it is the boundaries and not the regions of uniform intensity that provide information on movement, it is of interest to ask whether the two types of boundary, light-to-dark and dark-to-light, are used equally effectively by the bee's visual system to measure apparent angular speed. To examine this question, we used gratings whose spatial intensity profile was an asymmetrical sawtooth, as illustrated in Fig. 11a. With identical, stationary sawtooth gratings on the two walls, we find that bees always fly close to the axis of the tunnel, regardless of whether the gratings are oriented such that they offer sharp dark-to-light boundaries to both eyes of the flying bee (left panel, Fig. 11a), or sharp light-to-dark boundaries on one eye and sharp dark-to-light boundaries on the other (center and right panels, Fig. 11a). This finding indicates that sharp boundaries of a single polarity (either one) are sufficient to provide velocity information. However, it does not reveal whether bees are also capable of extracting this information from areas of the pattern where the intensity varies gradually, as in the ramp regions of the sawtooth profiles.

To investigate the latter question, we analyzed the trajectories of bees flying along the tunnel when both walls were lined with sinusoidal gratings (Fig. 11b). Again, we find that bees fly close to the axis of the tunnel, even when the spatial periods of the gratings on the two walls are unequal. It is only when the period of one of the gratings is reduced to $1.5 \mathrm{~cm}$ (corresponding to an angular period of $14 \mathrm{deg}$ for a bee flying along the axis) that there is a slight but statistically significant tendency for the bees to fly closer to that grating $(P<0.0005)$. In each of the cases shown in Fig. 11, motion of a grating causes the bees' flight trajectories to be displaced either toward or away from that grating, depending upon the direction of its motion in relation to that of the bee, as in the other experiments (data not shown). This set of results indicates that the bee's visual sys- tem can compute the apparent angular speed of a pattern accurately even when there are no sharp boundaries, and irrespective of the slopes of the intensity gradients in the pattern (see also Discussion).

\section{Dependence of the centering response on the contrast of the pattern}

The dependence of motion perception on pattern contrast was investigated by analyzing the trajectories of bees flying through the tunnel while one wall carried a stationary sinusoidal grating of a fixed, relatively high contrast, whilst the other carried a stationary sinusoidal grating of the same period, but whose contrast was varied from one experiment to the other. The results of experiments using two different combinations of contrasts are shown in Fig. 11c. The bees tend to fly close to the axis of the tunnel even when the contrasts of the gratings on the two walls are as disparate as 0.60 and 0.15 (Fig. 11c). It is only when the contrast of the pattern on one of the walls is very low $(<0.05)$ that the bees' trajectories begin to depart significantly from the axis of the tunnel. In fact, in conducting experiments in which one wall carries a high-contrast pattern and the other a zero-contrast pattern (i.e. a uniformly gray sheet), we find that considerable care has to be taken in producing and selecting the zero-contrast pattern because even small flaws, originating from spurious residual contrasts, are evidently sufficient to cause the flight trajectories to be located close to the tunnel axis. These findings indicate that the bee's perception of apparent angular speed is accurate even at very low contrast, and that the visual system is able to extract the apparent angular speed of a pattern largely independently of its contrast.

\section{Discussion}

This study, investigating the tendency of freely flying honeybees to fly along the axis of a tunnel, has demonstrated that flying honeybees balance the distances to the two walls by equalizing the apparent angular speeds on the two eyes. Whether image speed is translated into a true percept of range is a question that is not answered by the present investigation. There is, however, much circumstantial evidence to suggest that image speed is perceptually linked to object range in bees and other insects. Flying bees do not go near rapidly moving objects (see Srinivasan \& Lehrer, 1984a, and also below), suggesting that rapid image movement is interpreted as being caused by an object that is dangerously close. It has been shown that freely flying bees can be trained to distinguish between objects at different ranges, and that this discrimination is most likely mediated by monitoring image speed (Lehrer et al., 1988; Srinivasan et al., 1989). Locusts peer before they jump on to a nearby object, and are able to use the apparent motion of the object's image to estimate the object's range and adjust the size of their jump accordingly (Wallace, 1959; Sobel, 1990, 1991).

\section{The centering response vs. the optomotor response}

Several lines of evidence suggest that the centering response is mediated by motion-detecting mechanisms that are distinct from those that mediate the optomotor response in insects (reviews by Reichardt, 1987; Borst \& Egelhaaf, 1989): 

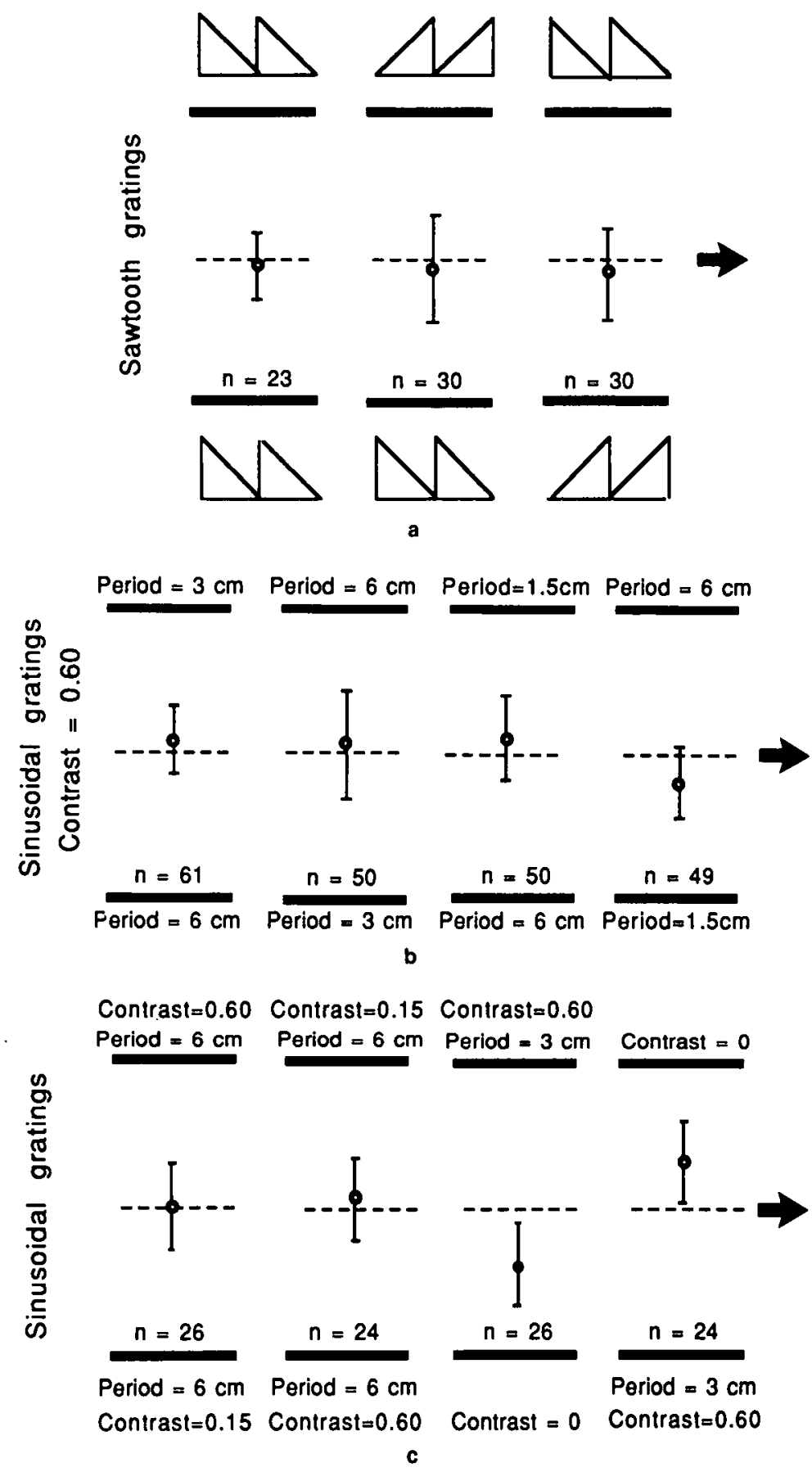

Fig. 11. Positions of trajectories (mean and standard deviation) of bees flying through the tunnel when the two walls present stationary patterns carrying a variety of intensity profiles and contrasts. (a) Sawtooth-profile gratings, with the abrupt intensity edges facing the same or opposite directions on the two walls. (b) Sinusoidal gratings of different periods on the two walls. (c) Sinusoidal gratings of equal periods, but different contrasts on the two walls. $n=$ number of flights, in the direction of the arrow, analyzed in each case.

1. The response is not one of yaw (i.e. rotation about a vertical axis); rather, it is one in which the flying insect shifts its position in the tunnel laterally. This is seen most clearly in the trajctories shown in Figs. 2d, 2e, and 8, where one of the walls carries no pattern. If these trajectories were governed by a classical yaw-controlling optomotor response, we would expect them to curve toward the wall carrying the pattern; what we find, instead, is that the trajectories are consistently displaced away from that wall. von Buddenbrock \& MollerRacke (1952) investigated the behavior of flies walking toward a light source along a tunnel lined with gratings on the side walls. When one wall carried a vertical grating and the other wall was blank, the flies turned toward the patterned wall, exhibiting a distinctly optomotor-like behavior. This is in contradiction to our findings with flying honeybees. Interestingly, however, we have observed that naive bees, just 
learning to enter the tunnel and find the reward, occasionally show a tendency to turn towards the wall carrying the pattern. It is possible that these bees are exhibiting an optomotor response, just like the flies, which is suppressed, as training proceeds, to reveal another visuomotor mechanism.

2. In many insects, including bees and flies, it is well-established that the optomotor response elicited by a moving grating depends primarily on the contrast frequency induced by the grating, and not on its apparent angular speed (reviews by Reichardt, 1987; Borst \& Egelhaaf, 1989). A contrast-frequency-dependent response has also been observed in studies of motion detection and ocular tracking in man (e.g. Kelly, 1979; Burr \& Ross, 1982; Gellman et al., 1990). Behavioral measurements of the optomotor response of the bee (Kunze, 1961) and measurements of the response of large-field motion-sensitive descending neurons in the bee (Ibbotson \& Goodman, 1990) reveal a bell-shaped response curve with a peak at a relatively low contrast frequency of $10 \mathrm{~Hz}$, and a half-width of approximately $10 \mathrm{~Hz}$. If the centering response were driven by the same motion-sensitive mechanisms as the optomotor response, then the data of Kunze (1961) and of Ibbotson and Goodman (1990) would predict that, in the tunnel, stationary gratings inducing contrast frequencies of, say, $2 \mathrm{~Hz}$ on one eye and $8 \mathrm{~Hz}$ on the other would produce substantially different motion signals in the two eyes, impairing the operation of the centering response. Contrary to this expectation, we see that the centering response operates perfectly under these conditions (see Fig. 9, left panel) as well as with a variety of other combinations of contrast frequencies, demonstrating that the visual mechanisms mediating this response do not confound angular speed with contrast frequency. Our data indicate that the centering response is driven by the apparent angular speed of the moving pattern, and is largely independent of the spatial structure of the pattern or the contrast frequencies that it induces, provided that the pattern carries a few vertically oriented features that can be used to measure angular speed in the horizontal plane. The visual mechanisms mediating the centering response must be capable of encoding unambiguously angular speeds ranging from $100-800 \mathrm{deg} / \mathrm{s}$, even if the contrast frequencies on the two sides differ by a factor of 10 (see Figs. 9 and 10). This is true for a domain of contrast frequencies ranging from a fraction of a cycle per second to at least $30 \mathrm{~Hz}$ (see Figs. 9 and 10). David (1982) has encountered mechanisms of movement perception that are qualitatively similar. He finds that fruitflies flying in a wind tunnel regulate their ground speed according to the apparent angular speed of the environment, which they are able to measure accurately irrespective of its spatial-frequency content. Land and Collett (1974) inferred the presence of neural mechanisms sensitive to angular velocity in their study of chasing behavior in flies.

3. Further evidence that contrast frequency does not play an important role in the centering response comes from the following consideration. We have already seen that motion of the pattern on one of the walls causes the bees' trajectories to shift away from the axis of the tunnel (Figs. 2, 4, 5, and 6). This shift alters the apparent angular speeds on the two eyes, but not the contrast frequencies: the latter depend only upon the speeds of the bee and the pattern, and are indepen- dent of the bee's position along the width of the tunnel. It therefore follows that the centering response cannot be mediated by monitoring the contrast frequencies on the two eyes, because these do not change when the bee shifts its lateral position.

4. There is some evidence (as already described under Results) to suggest that the centering response is mediated by movement-responsive mechanisms that are insensitive to the direction of apparent motion as seen by the eye. That is, apparent motion in the front-to-back direction is not distinguished from apparent motion in the back-to-front direction at the same angular speed. This is different from the optomotor response, which is clearly direction-sensitive, and which needs to be so if it is to fulfill its role of visual stabilization. This difference is, in itself, not compelling evidence against the participation of optomotor-like mechanisms in the centering response because a direction-insensitive response can readily be generated by summing the outputs of a pair of directionally sensitive movement detectors with opposite preferred directions. However, it is interesting in the light of recent findings with respect to range computation in the peering locust, which suggest the participation of direction-insensitive motion-detecting mechanisms (Sobel, 1990 a, 1990 b). It may well be that direction-insensitive mechanisms of movement detection offer a simple, as yet undiscovered, means of measuring apparent angular speed independently of image structure, which direction-sensitive mechanisms do not.

We believe that the centering response reflects an innate behavior that many locomoting insects use to maneuver through narrow gaps. To execute such maneuvers successfully, it would seem desirable to use a motion-perception system that is sensitive to angular speed, rather than contrast frequency or spatial structure: such a system would enable an insect to fly through the middle of a gap between two branches, for example, even if the textures of the bark on the two branches are substantially different.

\section{Relationship of the centering response to the movement avoidance response}

It is known that bees, trained to collect a reward at a grating, find it difficult to approach the grating when it is in motion (Srinivasan \& Lehrer, 1984a). It has also been shown previously that bees avoid movement, but not flicker (Srinivasan \& Lehrer, 1984b, 1985). Thus, the neural mechanisms underlying this "movement avoidance response" (MAR) must respond to true movement, and not to mere modulations of intensity in time. The MAR operates over a wide domain of contrast frequencies ranging from a few Hertz to $200 \mathrm{~Hz}$ (Srinivasan \& Lehrer, 1984a). It is conceivable that the centering response of bees flying through the tunnel in the present experiments is a consequence of balanced, oppositely directed MARs elicited by gratings on the two sides. At high contrast frequencies $(60-200 \mathrm{~Hz})$, the MAR is dependent on contrast frequency rather than angular speed. At lower contrast frequencies (0$20 \mathrm{~Hz}$ ), however, the MAR appears to depend on angular speed rather than contrast frequency (see Fig. 5, Srinivasan \& Lehrer, $1984 a$ ), a property that meshes well with that of the centering response. 


\section{Neural mechanisms for the measurement of angular speed}

We are yet to discover the neurons and processing mechanisms that are involved in the measurement of the apparent angular speed of the image. The so-called "optomotor" neurons are the most widely studied movement-sensitive neurons in the insect optic lobe (review by Hausen \& Egelhaaf, 1989). The response of these neurons to the transient movement of spots or bars is approximately proportional to angular speed, and roughly independent of the size or shape of the object (Collett \& King, 1975; Olberg, 1981; but see Horridge \& Marcelja, 1990). However, the steady-state response to a moving, periodic grating depends strongly upon the spatial structure of the grating (e.g. spatial frequency and contrast) and the temporal frequency that it induces, rather than upon its angular velocity (Eckert, 1980; review by Borst \& Egelhaaf, 1989; Horridge \& Marcelja, 1990). What is needed are neurons, with input field sizes of around 10 $\mathrm{deg}$, that measure local image speed independently of the structure or contrast of the image, over an angular-speed range of $100-800 \mathrm{deg} / \mathrm{s}$, and a contrast-frequency range of $1-30 \mathrm{~Hz}$. As far as we know, to this date there are no published reports of neurons satisfying all of these requirements.

A simple neural scheme for the measurement of local image speed is suggested in Fig. 12. The moving image (level A) is first converted to a binary image composed of two levels ("black" and "white"), by an array of neurons that possess high sensitivity to contrast and saturate at low contrasts. The binary neural image (level B), which moves at the same velocity as the original image, is then spatially low-pass-filtered by a subsequent array of neurons, resulting in a moving neural image (level $\mathrm{C}$ ) in which the abrupt edges of the binary image have been converted to ramps of constant slope. The speed of the image can then be monitored by measuring the rate of change of response at the ramps. Accordingly, the neural image at this level is temporally differentiated by an array of phasically responding neurons,
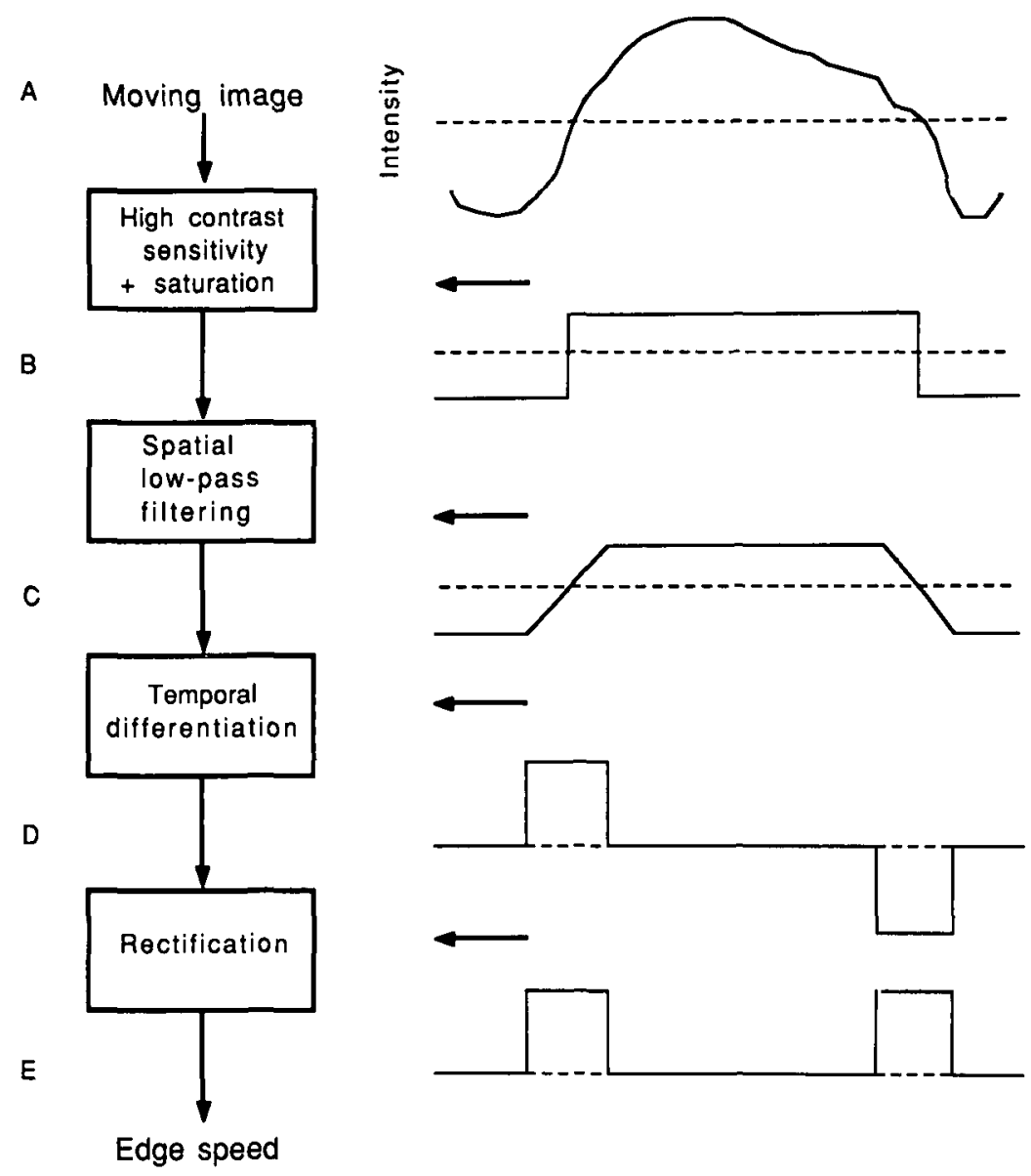

Fig. 12. A simple neural scheme proposed for measurement of the angular speed of an image, independently of its structure or contrast. The moving image (level A), whose spatial intensity profile at a given instant is as shown top right, is converted to a moving binary neural image (level B). The binary image is then spatially low-pass-filtered to yield a moving neural image in which the abrupt edges have been converted to ramps of constant spatial slope (level C). This neural image is then differentiated in time by an array of phasically responding neurons to yield a neural image composed of pulses (level D). The amplitudes of these pulses encode the local angular speed of the image. A subsequent stage of rectification ensures that angular speed is encoded independently of edge polarity or direction of motion. 
giving a moving neural image composed of a train of pulses (level D), one located at each edge of the binary image. The amplitude of each pulse will then be proportional to the rate of change of intensity at the corresponding ramp, and therefore to the instantaneous speed of the image at that location. A subsequent stage of rectification ensures that the response is positive, regardless of the polarity of the edge or the direction of movement (level E). Thus, we have a scheme that measures the local speed of the image, independently of structure, contrast, or direction of movement. A subsequent stage of lateral inhibition (not shown) would ensure that the system responds only to movement and not to flicker.

The scheme proposed here is, in effect, a modified version of the well-known gradient scheme that computes velocity as the ratio of the local temporal gradient to the local spatial gradient (Marr \& Ullman, 1981; Horn \& Schunck, 1981; review by Buchner, 1984; Fei Jin \& Srinivasan, 1990; Srinivasan, 1990). However, the present scheme has the advantage that it avoids the mathematical operation of division (and the attendant problems at low gradients, where the computation approaches the indeterminate value of zero/zero). It achieves this by parsing the image into edges and "standardizing" the spatial gradient to a constant, finite value at each edge.

The large monopolar cells (LMCs) in the lamina ganglion of the insect visual system are highly sensitive to moving edges, giving a response whose amplitude increases monotonically with edge speed over a range of $c a .0-1000 \mathrm{deg} / \mathrm{s}$ (Srinivasan et al., 1990), and with sinusoidal temporal frequency over a range of 0-30 Hz (Coombe et al., 1989). This property of the temporalfrequency response is mirrored closely by the MAR (Srinivasan \& Lehrer, 1984a). However, the responses of the LMCs are not entirely independent of the contrast of the edge. Neurons with dynamic properties similar to those of the LMCs, but which are less sensitive to variations of edge contrast, have been encountered recently by Dr. D. Osorio (personal communication) in the medulla of the locust. The response properties of these cells would correspond roughly to those at level D in the model of Fig. 12. Further work is needed to determine how accurately these medullary neurons encode image speed, and to ascertain whether they do indeed play a speed-encoding role.

\section{Acknowledgments}

Adrian Horridge, Michael Ibbotson, Daniel Osorio, and the anonymous reviewers provided many useful comments on the manuscript. We also thank Hans-Ortwin Nalbach for directing our attention to von Buddenbrock's early work. The computer-generated gratings were created using a program developed by GERT STANGE. Kym Witney's assistance in digitizing and analyzing bee flights was invaluable, as was Alex Saeck's generosity in providing and maintaining the bee colonies.

\section{References}

Borst, A. \& Egelhaaf, M. (1989). Principles of visual motion detection. Trends in Neurosciences 12, 297-306.

BUCHNER, E. (1984). Behavioral analysis of spatial vision in insects. In Photoreception and Vision in Invertebrates, ed. Aul, M.A., pp. 561621. New York: Plenum Press.

BurR, D.C. \& Ross, J. (1982). Contrast sensitivity at high velocities. Vision Research 22, 479-484.

Cheng, K., Collett, T.S., Pickhard, A. \& Wehner, R. (1987). The use of visual landmarks by honeybees: bees weight landmarks according to their distance from the goal. Journal of Comparative Physiology A 161, 469-475.

Collett, T.S. (1978). Peering - a locust behavior pattern for obtaining motion parallax information. Journal of Experimental Biology 76, 237-241.

ColleTt, T.S. (1988). How ladybirds approach nearby stalks: a study of visual selectivity and attention. Journal of Comparative Physiology $A$ 163, 355-363.

COLleTt, T.S. \& HARKNESS, L.I.K. (1982). Depth vision in animals. In Analysis of Visual Behavior, ed. Ingle, D.J., Goodale, M.A. \& Mansfield, R.J.W., pp. 111-176. Cambridge, Massachusetts: M.I.T. Press.

Collett, T. \& King, A.J. (1975). Vision during flight. In The Compound Eye and Vision in Insects, ed. HORRIDGE, G.A., pp. 437-466. Oxford: Clarendon Press.

Coombe, P.E., SRinivasan, M.V. \& GUY, R.G. (1989). Are the large monopolar cells of the insect lamina on the optomotor pathway? Journal of Comparative Physiology A 166, 23-35.

David, C.T. (1982). Compensation for height in the control of groundspeed by Drosophila in a new, "Barber's Pole" wind tunnel. Journal of Comparative Physiology 147, 485-493.

ECKERT, H. (1980). Functional properties of the HI-neurone in the third optic ganglion of the blowfly (Phaenicia). Journal of Comparative Physiology 135, 29-39.

ERIKSSON, E.S. (1980). Movement parallax and distance perception in the grasshopper Phaulacridium vittatum (Sjostedt). Journal of Experimental Biology 86, 337-340.

Fei Jin, Z. \& SRInivasan, M.V. (1990). Neural gradient models for the measurement of image velocity. Visual Neuroscience 5, 261-271.

Gellman, R.S., Carl, J.R. \& Miles, F.A. (1990). Short latency ocular following responses in man. Visual Neuroscience 5, 107-122.

Goulet, M., Campan, R. \& Lambin, M. (1981). The visual perception of relative distances in the wood-cricket (Nemobius sylvestris). Physiological Entomology 6, 357-367.

Hausen, K. \& EgelhaAf, M. (1989). Neural mechanisms of visual course control in insects. In Facets of Vision, ed. Stavenga, D.G. \& HARDIE, R.C., pp. 391-424. Berlin, Heidelberg: Springer-Verlag.

Horn, B.K.P. \& Schunck, B. (1981). Determining optical flow. Artificial Intelligence 17, 185-203.

HorRIDGE, G.A. (1986). A theory of insect vision: velocity parallax. Proceedings of the Royal Society $B$ (London) 229, 13-27.

Horridge, G.A. \& MARCELJA, L. (1990). Responses of the H1 neuron of the fly to contrast and moving bars. Philosophical Transactions of the Royal Society $B$ (London) 329, 75-80.

IBBotson, M.R. \& GoODMAN, L.J. (1990). Response characteristics of four wide-field motion-sensitive descending interneurones in Apis mellifera. Journal of Experimental Biology 148, 255-279.

KeLLY, D.H. (1979). Motion and vision, II: Stabilized spatio-temporal threshold surface. Journal of the Optical Society of America 69, 1340-1349.

KirChNER, W. \& SRINIVASAN, M.V. (1989). Freely flying honeybees use image motion to estimate object distance. Naturwissenschaften $\mathbf{7 6}$, $281-282$.

KUNZE, P. (1961). Untersuchung des Bewegungssehens fixiert fliegenden Bienen. Zeitschrift für vergleichende Physiologie 44, 656-684.

LAND, M.F. \& ColletT, T.S. (1974). Chasing behavior of houseflies (Fannia canicularis). Journal of Comparative Physiology 89, 331-357.

LEHRER, M. (1990). How bees use peripheral eye regions to localize a frontally positioned target. Journal of Comparative Physiology 167, 173-185.

Lehrer, M., Srinivasan, M.V., Zhang, S.W. \& Horridge, G.A. (1988). Motion cues provide the bee's visual world with a third dimension. Nature (London) 332, 356-357.

MarR, D. \& Ullman, S. (1981). Directional selectivity and its use in early visual processing. Proceedings of the Royal Society $B$ (London) 211, 151-180.

Olberg, R.M. (1981). Object and self-movement detectors in the ventral nerve cord of the dragonfly. Journal of Comparative Physiology 141, 327-334.

REICHARDT, W. (1987). Evaluation of optical motion information by motion detectors. Journal of Comparative Physiology 161, 533-547.

Sober, E.C. (1990a). Depth perception by motion parallax and paradoxical parallax in the locust. Naturwissenschaften 77, 241-243.

SOBEL, E.C. $(1990 b)$. The locust's use of motion parallax to measure distance. Journal of Comparative Physiology 167, 579-588.

SRINIVASAN, M.V. (1990). Generalized gradient schemes for the measurement of two-dimensional image motion. Biological Cybernetics 63, $421-431$. 
SrinivaSAN, M.V. \& Lehrer, M. (1984a). Temporal acuity of honeybee vision: behavioral studies using moving stimuli. Journal of Comparative Physiology A 155, 297-312.

Srinivasan, M.V. \& Lehrer, M. (1984b). Temporal acuity of honeybee vision: behavioral studies using flickering stimuli. Physiological Entomology 9, 447-457.

Srinivasan, M.V. \& Lehrer, M. (1985). Temporal resolution of color vision in the honeybee. Journal of Comparative Physiology 157 , 579-586.

Srinivasan, M.V. \& Lehrer, M. (1988). Spatial acuity of honeybee vision and its spectral properties. Journal of Comparative Physiology A 162, 159-172.
Srinivasan, M.V., Lehrer, M., Zhang, S.W. \& Horridge, G.A. (1989). How honeybees measure their distance from objects of unknown size. Journal of Comparative Physiology A 165, 605-613.

Srinivasan, M.V., Pinter, R.B. \& Osorio, D. (1990). Matched filtering in the visual system of the fly: large monopolar cells of the lamina are optimized to detect moving edges and blobs. Proceedings of the Royal Society $B$ (London) 240, 279-293.

VON BUdDENBROCK, W. \& MolleR-RACKe, L. (1952). Beitrag zum Lichtsinn der Fliege Eristalomyia tenax. Zoologischer Anzeiger 149, 51-61.

WALLACE, G.K. (1959). Visual scanning in the desert locust (Schistocerca gregaria Forskal). Journal of Experimental Biology 36, 512-525. 